\title{
Plasticity Like Model of Martensite Phase Transition in Shape Memory Alloys
}

\author{
S. Leclercq, G. Bourbon and C. Lexcellent \\ Laboratoire de Mécanique Appliquée R. Chaléat, URA 004 du CNRS, La Bouloie, Route de Gray, 25030 \\ Besançon cedex, France
}

\begin{abstract}
Many models simulating the behavior of Shape Memory Alloys (SMA) exist nowadays, in the one or three dimensional situation. In most of the cases they are not able to describe the non isothermal behavior of SMA due to the thermomechanical loadings. Indeed, the influence of the stress on the product phase leads to an orientation of the variants, when the temperature creates a self-accomodating martensite. The result of these two different influences is that the oriented product phase creates large deformations of phase transition, although the selfaccomodating one does not produce any macroscopical deformation.
\end{abstract}

In this paper, we develop a model of phase transition which takes into account the two types of martensite. Using this model allows us to simulate the behavior of SMA in isothermal or non isothermal loadings. Thus, the pseudoelasticity, the orientation of self-accomodating variants, the recovery stress or the thermal cycling at constant stress or strain can be simulated with a good agreement with experiments.

\section{INTRODUCTION}

The macroscopical modelling of the pseudoelastic effect of Shape Memory Alloys can be considered, at present time, as well known. A lot of papers devoted to ideal pseudoelasticity [1] on single crystals or on polycrystals $[2,3]$ have already fixed the ideas and made the problem clear.

Nevertheless, the behavior of SMA can not be reduced to the only pseudoelastic one ; and it could be interesting for users of SMA to be able to predict not only the isothermal, but the non isothermal behavior too.

We present, in this paper, a thermodynamical model allowing us to take into account the combined effect of the stress and of the temperature on the phase transition. Indeed, it is well known that the large macroscopical deformations characteristic to SMA are not due to the effect of temperature, which creates only self-accomodating martensite. This remark, first exposed by L.C. Brinson in [4], leads us to consider two types of martensite (the self-accomodating one and the oriented one), each having its own mass fraction ( $z_{T}$ and $z_{\sigma}$, respectively). We have to point out that this differentiation between the two martensites can be observed on micrographs performed by M.A. Morris [5].

Thus, the classical internal variable $\mathrm{z}$ (mass fraction of product phase) used in several models of pseudoelasticity, is here splitted into $\mathrm{z}_{\mathrm{T}}$ and $\mathrm{z}_{\mathrm{\sigma}}$. We show in next sections how adding one isotropic internal variable permits to take into account the general behavior of SMA. 


\section{THERMODYNAMICS AND CONSTITUTIVE HYPOTHESIS}

\subsection{Free energy of the system}

The model is written in the frame of the thermodynamics of irreversible processes, for the one dimensional situation. Thus, we postulate a specific free energy for the system :

$$
\psi=(1-z) \psi^{1}+z_{\mathrm{T}} \psi^{2}+z_{\sigma} \psi^{3}+\Delta \psi
$$

where : $0 \leq \mathrm{z}=\mathrm{z}_{\sigma}+\mathrm{z}_{\mathrm{T}} \leq 1$

$$
\begin{aligned}
& \alpha=1 \rightarrow \text { austenite } \\
& \alpha=2 \rightarrow \text { self-accomodating martensite } \\
& \alpha=3 \rightarrow \text { orienting martensite }
\end{aligned}
$$

$\psi^{\alpha}=$ specific free energy of $\alpha$-phase $(\alpha=1,3)$ :

$$
\psi^{\alpha}=u_{o}^{* \alpha}-T s_{o}^{* \alpha}+\frac{E}{2 \rho}\left(\varepsilon_{\alpha}^{e}\right)^{2}+C_{v}\left[\left(T-T_{0}\right)-T \ln \frac{T}{T_{0}}\right]
$$

$\mathrm{u}_{0}^{* \alpha}, \mathrm{s}_{\mathrm{o}}^{* \alpha}$ : internal energy, entropy of $\alpha$-phase at stress-free state and $\mathrm{T}=\mathrm{T}_{\mathrm{o}}$

$\varepsilon_{\alpha}^{e}:$ elastic strain of $\alpha$-phase

$\mathrm{E}$ : Young's modulus

$\rho$ : mass density

$T$ : current temperature

$T_{0}:$ reference temperature

$\mathrm{C}_{\mathrm{v}}$ : specific heat

The term $\Delta \psi$ is called coherency energy, and depends on interactions between the phases. We write :

$$
\Delta \psi=z_{\sigma}(1-z) \phi^{\mathrm{a \sigma}}+z_{\mathrm{T}}(1-z) \phi^{\mathrm{aT}}+z_{\sigma} z_{\mathrm{T}} \phi^{\sigma \mathrm{T}}
$$

where : $\phi^{\mathrm{a} \sigma}$ represents the interaction energy between phases 1 and 3

$\phi^{2 \mathrm{~T}}$ represents the interaction energy between phases 1 and 2

$\phi^{\sigma \mathrm{T}}$ represents the interaction energy between phases 2 and 3

For simplicity, and in accord with experiments, we assume :

$$
\begin{aligned}
& \phi^{\mathrm{a} \sigma}=\phi^{\mathrm{aT}}=\phi_{\mathrm{it}} \\
& \phi^{\sigma \mathrm{T}}=\hat{\phi}_{\mathrm{jt}}
\end{aligned}
$$

Substituting (4) into (3) and using $z=z_{\sigma}+z_{T}$ leads to :

$$
\Delta \psi=z(1-z) \phi_{i t}+z_{\mathrm{o}} z_{T} \hat{\phi}_{i t}
$$

\subsection{Constitutive hypotheses and behavior's laws}

We assume, as hypothesis 1 , that $\varepsilon_{1}^{e}=\varepsilon_{2}^{e}=\varepsilon_{3}^{e}=\varepsilon^{e}$, and $\varepsilon=\varepsilon^{e}+\varepsilon^{\mathrm{l}}$ is the macroscopical strain of the system.

The evolution law of the phase-transition deformation $\varepsilon^{\mathrm{tr}}$ is given by :

$$
\dot{\varepsilon}^{\mathrm{t}}=\gamma \dot{z}_{a}
$$


where $\gamma$ is the maximum deformation of phase transition in the one-dimensional situation. Equation (6) constitutes hypothesis 2 .

The behavior's equations read :

$$
\begin{aligned}
& \sigma=\rho \frac{\partial \psi}{\partial \varepsilon^{\mathrm{e}}}=\mathrm{E} \varepsilon^{\mathrm{e}} \\
& \varepsilon=\varepsilon^{\mathrm{e}}+\varepsilon^{\mathrm{tr}} \\
& \dot{\varepsilon}^{\mathrm{tr}}=\gamma \dot{z}_{\sigma}
\end{aligned}
$$

\subsection{The dissipation}

Taking into account the equations (7), one can write the intrinsic dissipation of the system, i.e, the second principle of thermodynamics.

$$
\pi_{\sigma}^{\mathrm{f}} \dot{\mathbf{z}}_{\sigma}+\pi_{\mathrm{T}}^{\mathrm{f}} \dot{\mathbf{z}}_{\mathrm{T}} \geq 0
$$

where :

$$
\left\{\begin{array}{l}
\pi_{\sigma}^{\mathrm{f}}=\frac{\gamma \sigma}{\rho^{\mathrm{f}}}-(1-2 \mathrm{z}) \phi_{\mathrm{it}}-\mathrm{z}_{\mathrm{T}} \hat{\phi}_{\mathrm{it}}+\pi_{\mathrm{o}}^{\mathrm{f}}(\mathrm{T}) \\
\pi_{\mathrm{T}}^{\mathrm{f}}=\pi_{\mathrm{o}}^{\mathrm{f}}(\mathrm{T})-(1-2 \mathrm{z}) \phi_{\mathrm{it}}-\mathrm{z}_{\sigma} \hat{\phi}_{\mathrm{it}}
\end{array}\right.
$$

are the thermodynamical forces associated to $\mathrm{z}_{\sigma}$, and $\mathrm{z}_{\mathrm{T}}$, respectively.

$$
\pi_{\mathrm{o}}^{\mathrm{f}}(\mathrm{T})=\Delta \mathrm{u}^{*}-\mathrm{T} \Delta \mathrm{s}^{*} \quad\left\{\begin{array}{l}
\Delta \mathrm{u}^{*}=\mathrm{u}^{*} \text { (parent phase) }-\mathrm{u}_{\mathrm{o}}^{*} \text { (product phase) } \\
\Delta \mathrm{s}^{*}=\mathrm{s}_{\mathrm{o}}^{*} \text { (parent phase) }-\mathrm{s}_{\mathrm{o}}^{*} \text { (product phase) }
\end{array}\right.
$$

\section{FLOW RULES AND PRINCIPLE OF THE MODEL}

Our model is built like a plasticity one. Thus, we define two yield surfaces : one for the direct phase transition, and one for the reverse phase transition. Note that by direct transition, one means one of the following cases :

, $\mathrm{z}_{\sigma}$ and/or $\mathrm{z}_{\mathrm{T}}$ grow

. $z_{\sigma}$ grows and $z_{T}$ decreases with $z$ being constant

By reverse transition, one means :

$\mathrm{z}_{\sigma}$ and/or $\mathrm{z}_{\mathrm{T}}$ decrease

\subsection{Yield surfaces and their compatibility with the Clausius-Duhem inequality}

Let us define :

$$
\bar{H}(x)=\left\{\begin{array}{lll}
0 & \text { if } & x \leq 0 \\
1 & \text { if } & x>0
\end{array} \quad \delta(x)=\left\{\begin{array}{lll}
0 & \text { if } & x \neq 0 \\
1 & \text { if } & x=0
\end{array} \quad \bar{\delta}(x)=\left\{\begin{array}{lll}
0 & \text { if } x=0 \\
1 & \text { overwise }
\end{array}\right.\right.\right.
$$

\subsubsection{Direct transition}

Let $f_{l}$ be the yield surface for direct phase transition :

$$
\mathrm{f}_{1}=\mathrm{f}_{1}^{\sigma} \overline{\mathrm{H}}(\|\bar{\sigma}\|)+\mathrm{f}_{1}^{\mathrm{T}} \overline{\mathrm{H}}(|\dot{\mathrm{T}}|)
$$


where :

$$
\begin{array}{lc}
f_{1}^{\sigma}=R_{1}^{\sigma}\left[\delta\left(R_{1}^{\sigma}\right)+\bar{\delta}\left(R_{1}^{\mathrm{T}}\right)\right] & R_{1}^{\sigma}=\pi_{\sigma}^{\mathrm{f}}-k_{\sigma}^{1}\left(z_{\sigma}\right) \\
f_{1}^{\mathrm{T}}=\mathrm{R}_{1}^{\mathrm{T}}\left[\delta\left(\mathrm{R}_{1}^{\mathrm{T}}\right)+\bar{\delta}\left(\mathrm{R}_{1}^{\sigma}\right)\right] & \mathrm{R}_{1}^{\mathrm{T}}=\pi_{\mathrm{T}}^{\mathrm{f}}-\mathrm{k}_{\mathrm{T}}^{1}\left(\mathrm{z}_{\mathrm{T}}\right) \\
\mathrm{k}_{\sigma}^{1}\left(\mathrm{z}_{\sigma}\right)=\frac{-\Delta \mathrm{s}^{*}}{\mathrm{a}_{\sigma}^{1}} \ln \left(1-\mathrm{z}_{\sigma}\right)+\left(4 \phi_{\mathrm{it}}-\hat{\phi}_{\mathrm{it}}\right) \mathrm{z}_{\sigma} \\
\mathrm{k}_{\mathrm{T}}^{1}\left(\mathrm{z}_{\mathrm{T}}\right)=\left(4 \phi_{\mathrm{it}}-\hat{\phi}_{\mathrm{it}}\right) \mathrm{z}_{\mathrm{T}}+\frac{\Delta \mathrm{s}^{*}}{\mathrm{a}_{\mathrm{T}}^{\mathrm{l}}}\left[\mathrm{b}_{1} \exp \left(-\mathrm{b}_{\mathrm{m}}^{\prime}\left(\mathrm{T}-\mathrm{M}_{\mathrm{s}}^{\mathrm{o}}\right)\right)-2\right] \ln \left(1-\mathrm{z}_{\mathrm{T}}\right)
\end{array}
$$

The consistency equation $\dot{f}_{1}=0$ gives the evolution rule of $z_{\sigma}$, assuming that $z_{T}$ evolves following the wellknown equation of Koistinen and Marburger $[5]\left(\dot{z}_{\mathrm{T}}=-\mathrm{a}_{\mathrm{T}}^{\mathrm{i}}\left(1-\mathrm{z}_{\mathrm{T}}\right) \dot{\mathrm{T}}\right)$.

\subsubsection{Reverse transition}

$\mathrm{f}_{2}$ is the yield surface of reverse transition:

$$
\mathrm{f}_{2}=\mathrm{f}_{2}^{\sigma} \overline{\mathrm{H}}(\| \bar{\sigma} \mid)+\mathrm{f}_{2}^{\mathrm{T}} \overline{\mathrm{H}}(|\dot{\mathrm{T}}|)
$$

where :

$$
\begin{array}{lc}
f_{2}^{\sigma}=R_{2}^{\sigma}\left[\delta\left(R_{2}^{\sigma}\right)+\bar{\delta}\left(R_{2}^{\mathrm{T}}\right)\right] & R_{2}^{\sigma}=-\pi_{\sigma}^{\mathrm{f}}+\mathrm{k}_{\sigma}^{2}\left(\mathrm{z}_{\sigma}\right) \\
\mathrm{f}_{2}^{\mathrm{T}}=\mathrm{R}_{2}^{\mathrm{T}}\left[\delta\left(\mathrm{R}_{2}^{\mathrm{T}}\right)+\bar{\delta}\left(\mathrm{R}_{2}^{\sigma}\right)\right] & \mathrm{R}_{2}^{\mathrm{T}}=-\pi_{\mathrm{T}}^{\mathrm{f}}+\mathrm{k}_{\mathrm{T}}^{2}\left(\mathrm{z}_{\mathrm{T}}\right) \\
\mathrm{k}_{\sigma}^{2}\left(\mathrm{z}_{\sigma}\right)=\left(4 \phi_{\mathrm{it}}-\hat{\phi}_{\mathrm{it}}\right)\left(\mathrm{z}_{\sigma}-1\right)+\frac{\Delta s^{*}}{\mathrm{a}_{\sigma}^{1}} \ln \mathrm{z}_{\sigma} \\
\mathrm{k}_{\mathrm{T}}^{2}\left(\mathrm{z}_{\mathrm{T}}\right)=\left(4 \phi_{\mathrm{it}}-\hat{\phi}_{\mathrm{it}}\right)\left(\mathrm{z}_{\mathrm{T}}-1\right)+\frac{\Delta s^{*}}{\mathrm{a}_{\mathrm{T}}^{2}}\left[2-\mathrm{b}_{2} \exp \left(-\mathrm{b}_{\mathrm{M}}\left(\mathrm{T}-\mathrm{A}_{\mathrm{s}}^{\circ}\right)\right)\right] \ln \mathrm{z}_{\mathrm{T}}
\end{array}
$$

In the same manner as for the previous section, $\dot{f}_{1}=0$ gives the evolution of $\dot{z}_{\sigma}$ and $\dot{z}_{T}\left(\dot{z}_{T}=-a_{T}^{2} z_{T} \dot{T}\right)$. The parameters introduced above $\left(a_{\sigma}^{1}, a_{\sigma}^{2}, a_{T}^{1}, a_{T}^{2}, b_{1}, b_{2}, b_{m}, b_{m}^{\prime}\right)$ are to be determined by experiments.

\subsubsection{Clausius-Duhem inequality}

An easy calculation using eq. (9) and (10) and the consistency equations proves that the second principle of thermodynamics is followed by our model in every thermomechanical loading.

\subsection{Principle of the model and results}

The model follows the rule hereafter exposed :

$$
\begin{aligned}
& \mathrm{f}_{\mathrm{i}}<0 \Rightarrow \text { no phase transition } \\
& \mathrm{f}_{\mathrm{i}}=0, \quad(\mathrm{i}=1,2) \\
& \mathrm{f}_{\mathrm{i}}=0, \dot{\mathrm{f}}_{\mathrm{i}}<0 \Rightarrow \text { phase transition } \\
& \mathrm{f}_{\mathrm{i}}>0 \text { is not possible }
\end{aligned}
$$

We have tested it on a $\mathrm{Cu} \mathrm{Zn} \mathrm{Al}\left(\mathrm{M}_{s}=40^{\circ} \mathrm{C}\right)$, for several thermomechanical loadings. Figure 1 to 6 show the results obtained by simulation, compared with the experimental ones. 


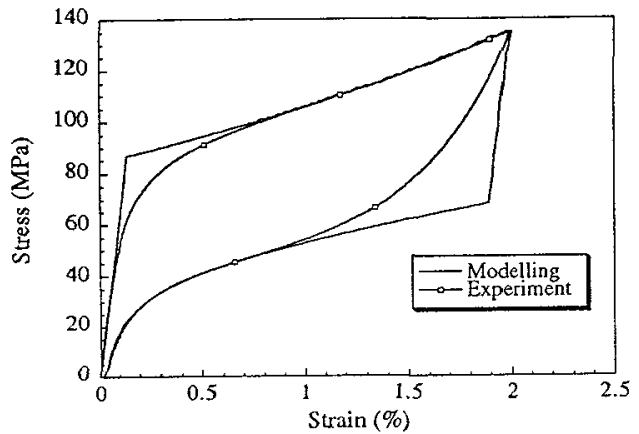

Fig. $1:$ Loading-unloading tensile test $\mathrm{Cu} \mathrm{Zn} \mathrm{Al}$. T $-\mathrm{M}_{\mathrm{s}}=16^{\circ} \mathrm{C}$

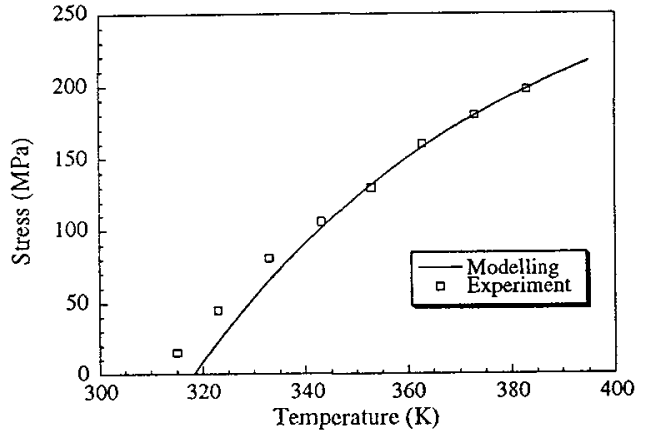

Fig. 2 : Recovery stress

$\mathrm{Cu} \mathrm{Zn} \mathrm{Al.} \varepsilon=1,15 \%$

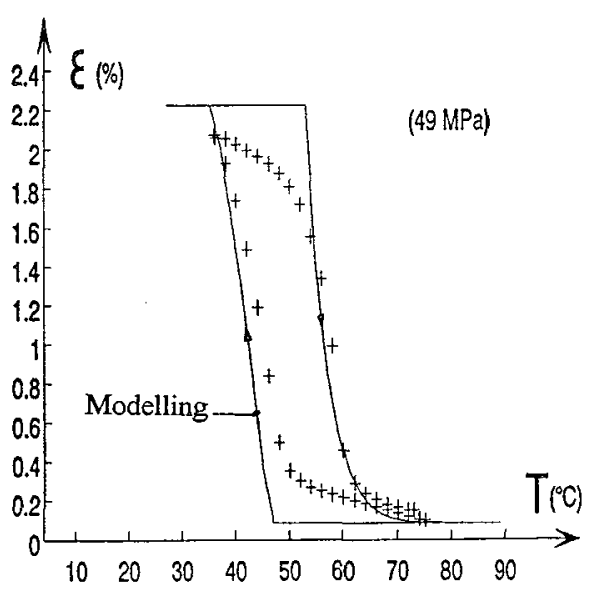

Fig. 3 : Thermal cycling $\mathrm{Cu} \mathrm{Zn} \mathrm{Al} . \sigma=49 \mathrm{MPa}$.

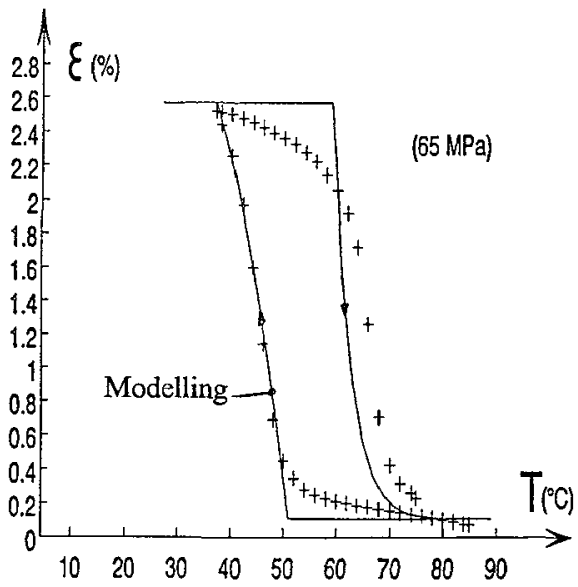

Fig. 4 : Thermal cycling $\mathrm{Cu} \mathrm{Zn} \mathrm{Al.} \sigma=65 \mathrm{MPa}$ 


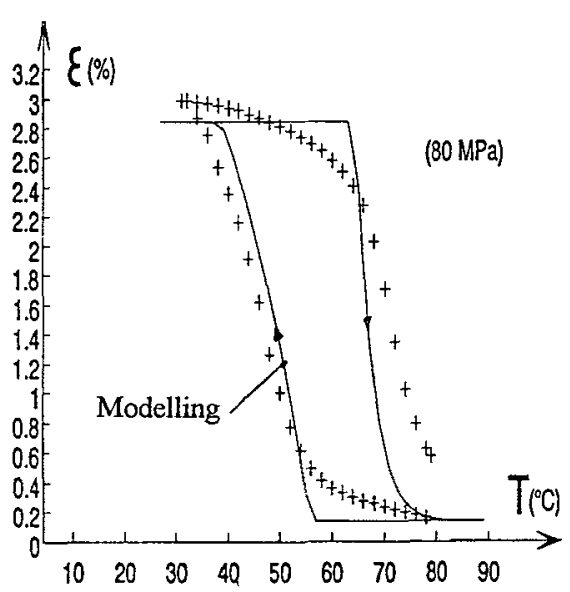

Fig. 5 : Thermal cycling

$\mathrm{Cu} \mathrm{Zn} \mathrm{Al.} \sigma=80 \mathrm{MPa}$

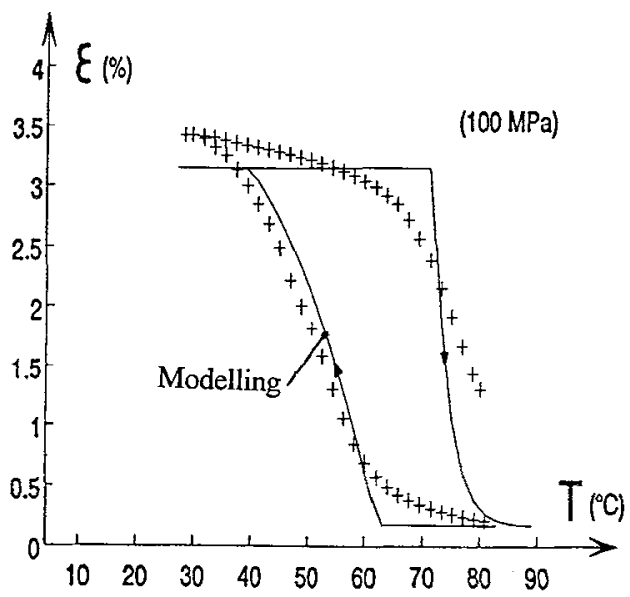

Fig. 6 : Thermal cycling $\mathrm{Cu} \mathrm{Zn} \mathrm{Al} . \sigma=100 \mathrm{MPa}$

\section{CONCLUSIONS}

The results given by the model exposed here are in good agreement with experiments. A future paper will develop it more in details, showing more simulations and how the internal loops are taken into account.

\section{REFERENCES}

[1] Huo Y. and Müller I., Continuum Mech. Thermodyn., 5, (1993), pp. 163-204

[2] Raniecki B. and Lexcellent C., Eur. J. Mech. A/Solids, 13, n 1, (1994), pp. 21-50

[3] Abeyaratne R. and Knowles J.K., J. Mech. Phys. Solids, 41, n³, (1993), pp. 541-571

[4] Brinson L.C., Journal of Intelligent Systems and Structures, (1991), pp. 275-309

[5] Morris M.A., Joint work between LMA (Besançon) and Inst. of Struc. Metal (Neuchâtel), private communication

[6] Koistinen D.P. and Marburger R.E., Acta Met., 7, (1959), pp. 59-60 